\title{
Design Principles of Wind Turbine Inertia Emulators for Autonomous Systems
}

\author{
Lampros N. Papangelis*. Costas D. Vournas* \\ *Department of Electrical and Computer Engineering, \\ National Technical University of Athens, Zografou, Greece \\ (e-mail:labros.papangelis@gmail.com; vournas@power.ece.ntua.gr)
}

\begin{abstract}
The problem of frequency control and stability has become more prominent with the increasing use of converter-connected generators in many countries around the world. Especially in small autonomous systems, the replacement of conventional synchronous generation by variable-speed wind turbines may lead to large deviations of system frequency after severe transients and possibly even customer disconnection. For wind generators, a control scheme that is already being implemented, in order to mitigate this problem, is the addition of an inertia emulator. This paper proposes a design principle for wind turbine inertia emulators based on the characteristics of the autonomous system to which the wind turbines are connected.
\end{abstract}

Keywords: Wind Turbine Generators, inertia emulator, autonomous system, variable-speed wind generators, frequency control, frequency stability.

\section{INTRODUCTION}

Constantly increasing penetration of wind turbines in electric power systems poses various technical challenges as the increase of wind generation leads to replacement of conventional synchronous generators. Concerning system frequency control, the rotor speed (and inertia) of variable-speed wind turbine generators (WTG) with doubly fed asynchronous generators (DFAG) or full converter wind generators (FCWG) is decoupled from the power system frequency, thus these generators provide negligible inertia response (Lalor et al., 2004). As a result, the total system inertia decreases leading to increased rate of change of frequency, especially in autonomous systems, and possibly load shedding by underfrequency (or rate-of-change-of-frequency) relays after large power disturbances (Lalor et al., 2005).

To counteract this potential problem, variable-speed wind turbines can be equipped with an additional supplementary control loop in order to provide inertial response to the system. Control schemes have been proposed in the literature for variable-speed wind turbines, in order to emulate inertial behavior of conventional generating units (Marinelli et al., 2011; Morren et al., 2006; Gautan et al., 2011, Ruttledge et al., 2012). A simple implementation of the Inertia Emulation Controller (IEC) consists of a washout filter and a gain, as shown in Fig. 1. This emulator uses the accumulated kinetic energy in the wind turbine shaft to provide a temporary increase of the active power injected to the grid, proportional to the system frequency rate of drop. The emulator is added to the active power order signal provided by the maximum power tracking controller of the wind turbine.

Inertia emulation is commercially available today. However, it is a quite recent feature, for the proper tuning of which further research is justified. In this paper a generic autonomous system model is used to demonstrate the quantitative increase in system inertia offered by the inertia emulator. Based on the same model a general rule is derived in order to choose the washout time constant of Fig. 1, based upon the dominant frequency regulation loop time constant of the autonomous system. The controller gain is set in all cases using simulation, as it depends heavily on the nonlinear response of the system. The interaction of the IEC with wind turbine dynamics is also investigated in detail in the paper including the effect of energy recovery after the initial rotor deceleration, and certain controller adjustments are proposed to improve the WTG performance with the IEC.

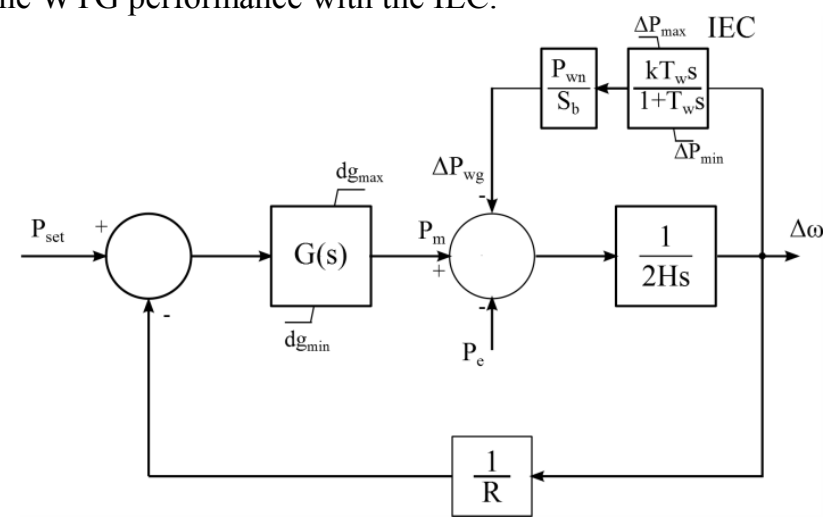

Fig. 1 Generic frequency control loop with inertia emulator

\section{DESIGN PRINCIPLE}

\subsection{Generic Autonomous System Model}

In this Section the effect of the inertia emulator on a simple generic model of the frequency regulation loop of an autonomous system (Fig.1) is examined in order to propose a design principle for Inertia Emulation Controllers (IEC). All the 
conventional power plants are considered to have the same technical characteristics (e.g. speed governors and prime movers) and are aggregated in a single equivalent unit model. The rate of change of system frequency near its nominal value is then given by:

$$
P_{m}-P_{e}=2 H \dot{\omega}
$$

where $P_{m}$ is the sum of mechanical powers of all the prime movers, $P_{e}$ the electrical demand not covered by WTGs and $\mathrm{H}$ the inertia constant of the equivalent conventional power plant, with all per unit values referred to the system base power $S_{b}$. Wind turbine dynamic behavior is not modeled in Fig. 1, where IEC is represented as a direct power injection assuming instantaneous WTG response. Similarly to the conventional plants, a single aggregate WTG with a total rating of $P_{w n}$ is assumed.

The open loop $(R \rightarrow \infty)$ transfer function (OLTF) of the autonomous system without the inertia emulator is:

$$
G_{o, n}(s)=\frac{1}{2 H s(1+T s)}
$$

When a large power disturbance occurs, the rate of change of frequency is proportional to the term $1 / 2 \mathrm{H}$ and the dominant pole of the governor/prime mover OLTF is $-1 / T$.

\subsection{Effect of Inertia Emulator}

When the inertia emulator is introduced as in Fig. 1, the open loop transfer function is modified as follows:

$G_{o, n}(s)=\frac{1+T_{w} s}{\left(2 H+k^{\prime} T_{w}\right) s\left(1+\frac{2 H T_{w}}{2 H+k^{\prime} T_{w}} s\right)(1+T s)}$

where $k^{\prime}=k P_{w n} / S_{b}$ is the IEC gain referred to system basis.

Using (3) the added inertia by the IEC is clearly defined as the term $k^{\prime} T w$ added to system inertia $2 H$.This allows a quantitative assessment of the inertia added by the IEC. Also one pole and one zero are introduced in the OLTF (2).

\subsection{Selection of IEC time constant $T_{w}$}

The design principle proposed in this paper is to choose the value of the time constant of the wash-out filter to be equal to the dominant time constant of the system $\left(\mathrm{T}_{\mathrm{w}}=\mathrm{T}\right)$. In this way, the zero of the OLTF (3) cancels the dominant pole. At the same time, a new pole is introduced to the left of the original one at $-\left(1 / T+k^{\prime} / 2 H\right)$. A smaller value $T_{w}<T$ will not help, as the dominant pole will remain, whereas a larger value $T_{w}>T$ will simply make the emulator slower without any benefit.

With the suggested setting of $T_{w}=T$, the OLTF has exactly the same form with (2), with the inertia $2 H$ increased by the amount $k^{\prime} T_{w}$ (referred to system base power). The root locus of the frequency control loop of the generic system of Fig. 1 for increasing governor gain $K=1 / R$ is presented in Fig. 2 . The blue colored locus (closer to the imaginary axis) corre- sponds to the system without inertia emulator $(k=0)$, whereas the red colored one corresponds to $k=10$. As seen by comparing the closed loop poles for $R=0.05$ indicated by the two arrows in Fig. 2, the inertia emulator with the proposed design enhances significantly the stability and performance of the frequency control loop.

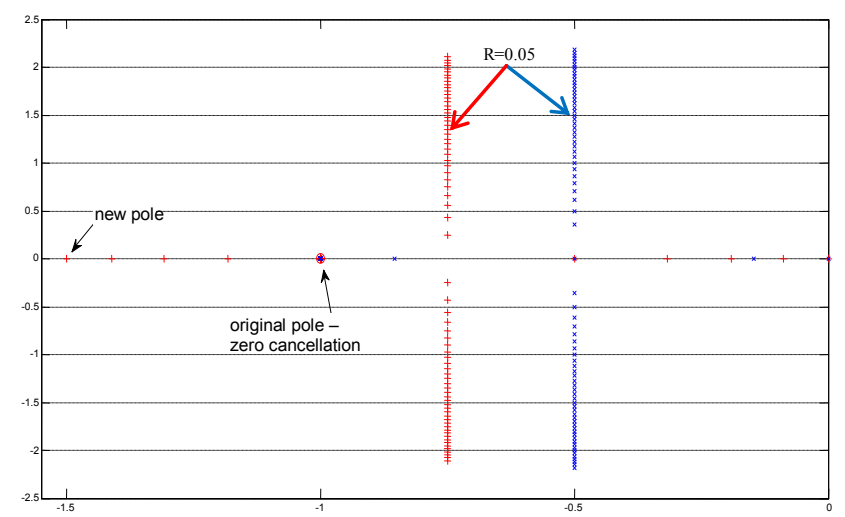

Fig. 2 Root locus for varying droop gain K (generic model)

\subsection{Calculation of maximum frequency drop}

In this subsection we derive analytic expressions for the variation of frequency versus time for a given power disturbance $\Delta P_{e}$. This calculation is important, because it provides the maximum frequency drop (and thus information concerning underfrequency load shedding) without resorting to simulation. From Fig. 1 the closed loop transfer function without IEC is:

$$
\frac{\Delta \Omega(s)}{\Delta P_{e}(s)}=-\frac{T s+1}{2 H s(T s+1)+1 / R}
$$

The roots of the denominator of (4) are:

$$
s_{1,2}=-\sigma \pm j \omega_{d}=-\frac{1}{2 T} \pm j \frac{\sqrt{\frac{8 H T}{R}-4 H^{2}}}{4 H T}
$$

assuming $2 T / R>H$, which normally holds as $R$ is usually very small (typically between 0.02 and 0.05 ).

By applying Inverse Laplace transform on (4) the variation of system frequency versus time is calculated as follows:

$$
\begin{aligned}
\Delta \omega(t)=-\Delta P_{e}[R & -R e^{-\sigma t} \cos \left(\omega_{d} t\right) \\
& \left.+\frac{1-2 H \sigma R}{2 H \omega_{d}} e^{-\sigma t} \sin \left(\omega_{d} t\right)\right]
\end{aligned}
$$

From (6), the time that the system frequency becomes minimum (for a positive $\Delta P_{e}$ ) can be calculated as:

$$
t_{\text {minf }}=\frac{1}{\omega_{d}}\left[\pi+\arctan \left(-\frac{\omega_{d}}{2 H \omega_{d}^{2} R-\sigma+2 H \sigma^{2} R}\right)\right]
$$


By substituting $t_{\operatorname{minf}}$ to (6), the maximum frequency drop is readily derived.

The same method is used to calculate the maximum frequency drop after the introduction of the proposed inertia emulator with $T_{w}=T$. In this case, the transfer function (TF) of the frequency system after a disturbance $\Delta P_{e}$ is:

$$
\Delta \Omega^{\prime}(s)=-\Delta P_{e} \frac{T_{w} s+1}{\left(2 H+k^{\prime} T_{w}\right) s\left(\frac{2 H T_{w}}{2 H+k^{\prime} T_{w}} s+1\right)+\frac{1}{R}}(8)
$$

The roots of the denominator of (8) are:

$$
\begin{aligned}
s_{1,2}^{\prime} & =-\sigma^{\prime} \pm j \omega_{d}^{\prime} \\
& =-\frac{2 H+T k^{\prime}}{4 T H} \pm j \frac{\sqrt{\frac{8 H T}{R}-\left(2 H+k^{\prime} T_{w}\right)^{2}}}{4 H T}
\end{aligned}
$$

Comparing (5) and (9) it becomes evident that $\sigma^{>}>\sigma$ and $\omega_{d}{ }^{\prime}<\omega_{d}$, thus the damping ratio $\zeta$ increases with the IEC.

Again, the time instant of maximum frequency excursion is calculated by (7) with $\sigma$ and $\omega_{d}$ substituted by $\sigma^{\prime}$ and $\omega^{\prime}{ }_{d}$.

\subsection{Simulation Results}

The generic system is simulated for typical parameter values. All simulations were run in Simulink using integration routine "ode $15 \mathrm{~s}$ " with a variable step size and a relative tolerance equal to $10^{-3}$.

The frequency of the system for a $20 \%$ step increase of the load is shown in Fig. 3. It can be seen that with the introduction of the inertia emulator the maximum deviation of the frequency is decreased and the damping of the oscillations has increased.

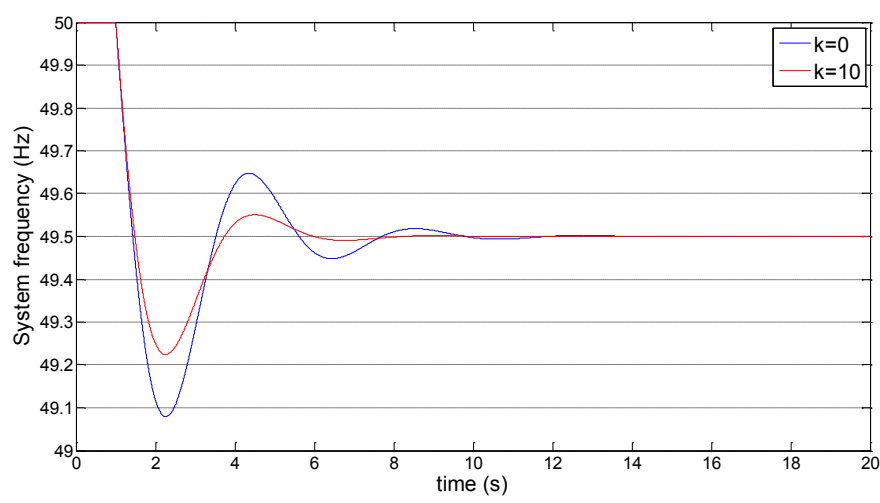

Fig.3 System frequency with and without inertia emulator

The eigenvalues of the generic system with and without IEC can be obtained from Fig. 2 for $R=0.05$ and are equal to $\mathrm{s}_{1,2}=-0.5 \pm \mathrm{j} 1.5$ and $\mathrm{s}_{1,2}=-0.75 \pm \mathrm{j} 1.392$ respectively. Using (6) and (7) the minimum frequency is found equal to $\mathrm{f}_{\min }=49.08 \mathrm{~Hz}$ without IEC $(k=0)$ and $\mathrm{f}_{\min }=49.22 \mathrm{~Hz}$ with IEC $(k=10)$. These values match exactly the results of the simulation shown in Fig. 3.

\section{APPLICATION TO SIMPLE SYSTEMS}

In this Section, the above method for selecting $T_{w}$ is applied on simple systems consisting of a constant power load and an aggregated generating unit. Two types of governors/prime mover models are examined: a fast unit (e.g. a Diesel generator) and a slower prime mover (e.g. a three-stage steam turbine).

For each system $T_{w}$ is chosen equal to the dominant time constant and the IEC design is completed by selecting the value of the gain $k$. The selection is based on simulation, as it depends on nonlinear dynamics, such as the effect of limiters. Specifically, the gain $k$ is gradually increased until the point, after which no further significant improvement can be achieved on the system frequency response after a severe disturbance. In this way it can be ensured that the wind turbines will transiently provide the highest possible active power for the most severe credible contingency (i.e. the loss of the largest power plant).

The inertia emulator uses the accumulated energy in the wind turbine shaft, in order to temporarily increase the active power production of the wind turbine. In order to avoid large excursions from the optimal steady-state operating point, an upper limit is set to the power increase caused by the inertia emulator. Due to the negative sign of the $\Delta \mathrm{P}_{\mathrm{WG}}$ signal in Fig. 1 this limit appears as a lower limit $\Delta P_{\text {min }}$, which is set to $10 \%$ (referred to the wind turbine nominal power) in this paper. Moreover, the inertia emulator is assumed to respond only to underfrequency events and should not be used to temporarily decrease the active power of the wind turbine for an overfrequency event (e.g. loss of load). Thus, the upper limit $\Delta P_{\max }$ is taken equal to 0 . Hereafter, when reference is made to the maximum output of the IEC, this relates to its absolute value.

\subsection{System with Fast Conventional Units}

The governor/prime mover TF in the case of a system with fast conventional units is:

$$
G(s)=\frac{\Delta P}{\Delta g}=\frac{1}{\left(1+T_{g} s\right)(1+T s)}
$$

where $T$ is the time constant of the valve opening mechanism and $T_{g}$ is the governor time constant.

The parameters of the fast conventional unit are given in Table 1 . Assuming the load is equal to $1 \mathrm{pu}$ on the system base, the conventional unit covers $76 \%$ of the overall load in the initial dispatch and the remaining is covered by the aggregated wind turbine $(0.24 \mathrm{pu})$, which corresponds to $60 \%$ of the WTG rating $(0.4 \mathrm{pu})$. The WTG is again considered as a constant power injection to the common bus modulated by the IEC. According to the analysis of the previous section, the time constant of the inertia emulator is chosen equal to the dominant time constant, thus in this case $T_{w}=0.2 \mathrm{~s}$. 
Table 1: Fast system parameters

\begin{tabular}{|c|c|c|c|}
\hline Parameter & Value & Parameter & Value \\
\hline $\mathrm{f}_{\mathrm{n}}$ & $50 \mathrm{~Hz}$ & $\mathrm{dg}_{\max }$ & $0.15 \mathrm{pu} / \mathrm{s}$ \\
\hline $\mathrm{S}_{\mathrm{b}}$ & $100 \mathrm{MVA}$ & $\mathrm{P}_{0}$ & $0.76 \mathrm{pu}$ \\
\hline $\mathrm{T}_{\mathrm{g}}$ & $0.1 \mathrm{~s}$ & $\mathrm{P}_{\mathrm{wn}}$ & $0.4 \mathrm{pu}$ \\
\hline $\mathrm{T}$ & $0.2 \mathrm{~s}$ & $\mathrm{P}_{\mathrm{w} 0}$ & $0.24 \mathrm{pu}$ \\
\hline $\mathrm{R}$ & 0.05 & $\Delta \mathrm{P}_{\min }$ & $-0.10 \mathrm{pu}$ \\
\hline $\mathrm{H}$ & $3.82 \mathrm{~s}$ & $\Delta \mathrm{P}_{\max }$ & $0 \mathrm{pu}$ \\
\hline
\end{tabular}

The disturbance considered takes place at $t=5 \mathrm{~s}$ and is simulated as a step increase of the load active power by $20 \%$. Fig. 4 shows the response of system frequency for increasing values of the gain $k$ of the inertia emulator $(k=0$ corresponds for no inertia emulator). The increase of $k$ up to 40 results in better response of the system frequency and a higher frequency nadir. However, further increase of the gain does not cause any improvement because the inertia emulator has quickly reached its maximum output limit of $10 \%$. Thus, the value $k=40$ is chosen for the gain of the IEC. It is noted that, due to the small value of $T_{w}$, a reasonable value of the overall gain $k T_{w}$ is less than $8 \mathrm{~s}$ (on WTG base).

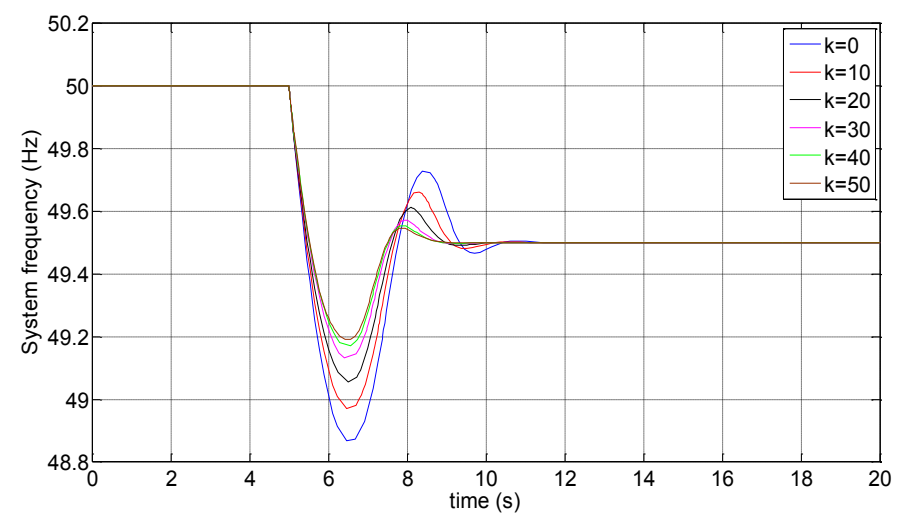

Fig. 4 System frequency for increasing $k$ (fast system)

\subsection{Slow Conventional Unit}

The same process is followed to determine the effect of the inertia emulator to a system where the conventional unit is a slower, three-stage steam turbine represented by the following TF with the data of Table 2:

$$
G(s)=\frac{1+\left[K_{h p} T_{r}+\left(1-K_{l p}\right) T_{l p}\right] s+K_{h p} T_{l p} T_{r} s^{2}}{\left(1+T_{g} s\right)\left(1+T_{h p} s\right)\left(1+T_{l p} s\right)\left(1+T_{r} s\right)}
$$

Compared to the fast unit of the previous subsection, the steam turbine involves three stages (high pressure, reheat and low pressure stage), each with a different time constant $\left(T_{h p}\right.$, $T_{r}, T_{l p}$ respectively). $T_{g}$ is again the time constant of the governor. The percentages of mechanical power produced by the high and low pressure stages are given by $K_{h p}$ and $K_{l p}$ respectively. The remaining percentage is produced by the reheat stage.

The same load is considered and the same disturbance is simulated. In order to avoid unstable frequency oscillations (Vournas, 1999), the rate limit of the steam turbine gate opening $\mathrm{dg}_{\max }$ is taken equal to the one of the fast unit of the previous subsection 3.1 .
Table 2: Slow system parameters

\begin{tabular}{|c|c|c|c|}
\hline Parameter & Value & Parameter & Value \\
\hline $\mathrm{f}_{\mathrm{n}}$ & $50 \mathrm{~Hz}$ & $\mathrm{R}$ & 0.05 \\
\hline $\mathrm{S}_{\mathrm{b}}$ & $100 \mathrm{MVA}$ & $\mathrm{H}$ & $3.82 \mathrm{~s}$ \\
\hline $\mathrm{T}_{\mathrm{g}}$ & $0.1 \mathrm{~s}$ & $\mathrm{dg}_{\max }$ & $0.15 \mathrm{pu} / \mathrm{s}$ \\
\hline $\mathrm{T}_{\mathrm{hp}}$ & $0.2 \mathrm{~s}$ & $\mathrm{P}_{0}$ & $0.76 \mathrm{pu}$ \\
\hline $\mathrm{T}_{\mathrm{r}}$ & $4 \mathrm{~s}$ & $\mathrm{P}_{\mathrm{wn}}$ & $0.4 \mathrm{pu}$ \\
\hline $\mathrm{T}_{\mathrm{lp}}$ & $0.3 \mathrm{~s}$ & $\mathrm{P}_{\mathrm{w} 0}$ & $0.24 \mathrm{pu}$ \\
\hline $\mathrm{K}_{\mathrm{hp}}$ & 0.4 & $\Delta \mathrm{P}_{\min }$ & $-0.10 \mathrm{pu}$ \\
\hline $\mathrm{K}_{\mathrm{lp}}$ & 0.3 & $\Delta \mathrm{P}_{\max }$ & $0 \mathrm{pu}$ \\
\hline
\end{tabular}

The time constant of the inertia emulator is chosen to be equal to the time constant of the reheat stage of the steam turbine, which is the dominant time constant in this case $\left(T_{w}=4 \mathrm{~s}\right)$. In Fig. 5 the response of system frequency is shown. The frequency drop without the inertia emulator $(k=0)$ is much more pronounced than in the fast system and would probably lead to underfrequency load shedding, in order to avoid the collapse of the system. It can be seen that as the gain $k$ increases, the response of the system frequency greatly improves. More specifically, the oscillations are damped faster and the frequency nadir for $k \geq 4$ is around $48.5 \mathrm{~Hz}$.

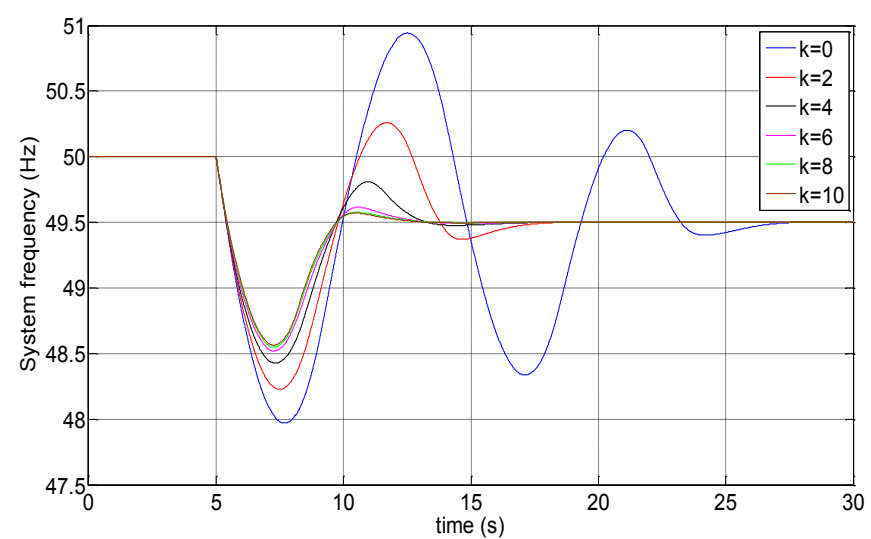

Fig. 5 System frequency for increasing $k$ (slow system)

As in the previous system, the increase of gain $k$ does not always result in better response because of the saturation in the output of the inertia emulator. In this case the value $k=6$ is chosen.

As shown, the addition of the inertia emulator with the proposed design principle has similar effect on both the fast and slow system. In the following sections only the slow system, for which the effect of the disturbance is most severe, will be considered.

\section{EFFECT OF WIND TURBINE DYNAMICS}

\subsection{Modeling and Initial Operating Point}

The DFAG and FCWG models used in this section have been presented in Tsourakis et al. (2009) and Nanou et al. (2011) respectively. The inertia emulator of Fig. 1 is introduced to these models, which are then added to the two systems of the previous section. Only the responses of the slow system are reported in this Section. 
Two cases of wind turbine initial loading conditions are examined for each WTG model as shown in Table 3: partial load $(60 \%$ of the nominal active power) with the pitch angle limited to its minimum value and nominal load with wind speed above nominal and positive initial pitch angle $\beta>0$. The initial steam turbine active power is adjusted in every case so that the pre-disturbance sum of WTG active power and steam turbine active power is equal to 1 .

Table 3: WTG nominal and generated active power

\begin{tabular}{|c|c|c|c|}
\hline WTG model & $\mathbf{P}_{\mathbf{w n}}(\mathbf{p u})$ & $\mathbf{P}_{\mathbf{w 0}}(\mathbf{p u})$ & $\mathbf{P}_{\mathbf{m} \mathbf{0}}(\mathbf{p u})$ \\
\hline$(\beta=0)$ & 0.4 & 0.24 & 0.76 \\
\hline$(\beta>0)$ & 0.4 & 0.4 & 0.6 \\
\hline
\end{tabular}

\subsection{DFAG Wind Turbine $(\beta=0)$}

For the slow system of Section 3.2, with selected IEC time constant $T_{w}=4 \mathrm{~s}$ the same process as in Section 3 is followed to determine the value of the emulator gain $k$ for the DFAG wind turbine. Figure 6 shows the system frequency for increasing inertia emulator gain.

As can be seen in Fig. 6 the effect of the inertia emulator is qualitatively similar to that without wind turbine dynamics (Fig. 5), with the increase of the gain $k$ resulting in improved response until the effect of saturation due to IEC output limiter is noticed. The value of emulator gain chosen is $k=4$. As seen, the improvement achieved is not as significant as in the case without the wind turbine dynamics. This is due to the effect of the wind turbine internal control loops as explained below:

The increased active power output due to IEC causes the wind turbine rotor to decelerate as shown in Fig. 7. It should be noted at this point that the decrease of the aerodynamic power due to the reduced aerodynamic coefficient is not taken into account in the simulation. However, as shown in Fig. 7, the rotor speed deviation from the initial value is very small (in the order of $1 \%$ ), and therefore the effect on the aerodynamic power is considered negligible.

Following the deviation of the rotor speed the torque control of the wind turbine decreases accordingly the power order signal ( $\mathrm{P}_{\text {ord }}$ in Fig. 8), and thus cancels out the effect of the IEC. This results in reduced inertia support from the DFAG and a decrease of the active power output, even below the initial value as shown in Fig. 9.

In order to mitigate this inadvertent effect on the inertia emulation, the parameters of the DFAG torque controller are retuned. The values of the proportional and integral gain of the PI controller shown in Fig. 8 are reduced from $k_{\text {ptrq }}=3$ to $k_{p t r q}=1$ and from $k_{i t r q}=0.6$ to $k_{i t r q}=0.2$ respectively. Also, the value of the time constant $T_{p c}$ is increased to $4 \mathrm{~s}$ from $0.05 \mathrm{~s}$.

With this modification, the DFAG speed controller becomes less sensitive to the deviation of the rotor speed and the decrease of the $P_{\text {ord }}$ signal becomes less aggressive. In Fig. 10 the responses of the system frequency for the initial and the new speed control parameters, as well as the one with the ideal inertia emulator (no wind turbine dynamics) are compared for $T_{w}=4 \mathrm{~s}$ and $k=4$. The above modification of speed control parameters does not affect adversely the normal speed control for variable wind speed, since the wind rotor speed returns to its initial value in less than $30 \mathrm{~s}$, as can be seen in Fig. 10.

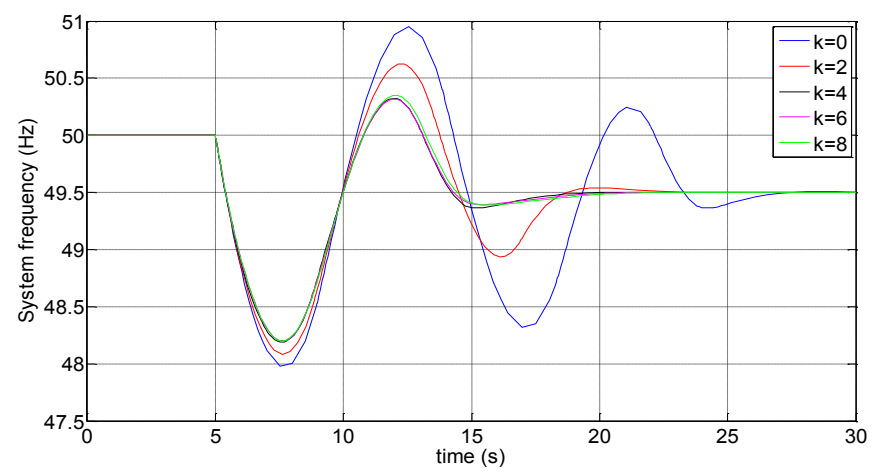

Fig. 6 System frequency for increasing gain $k$

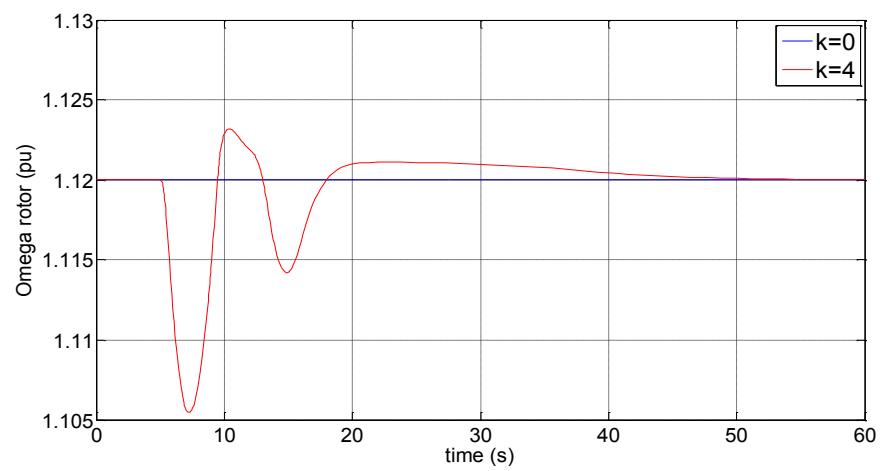

Fig. 7 DFAG rotor speed with and without inertia emulator
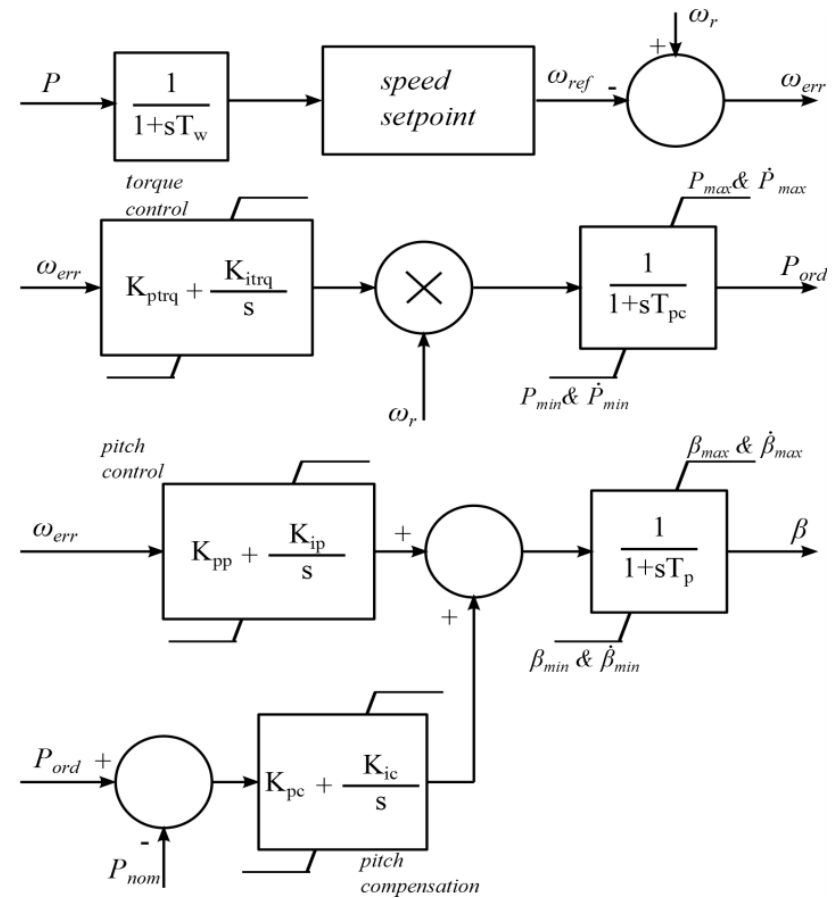

Fig. 8 DFAG speed and pitch controller 


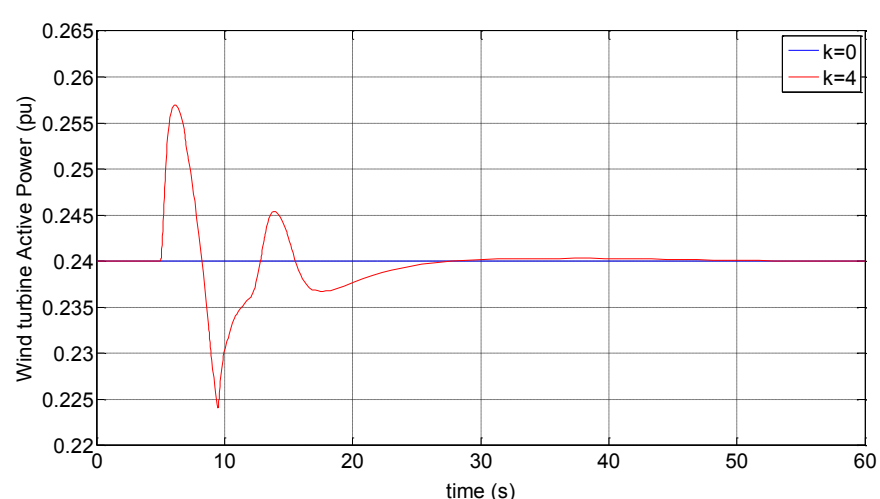

Fig. 9 DFAG active power output with and without inertia emulator

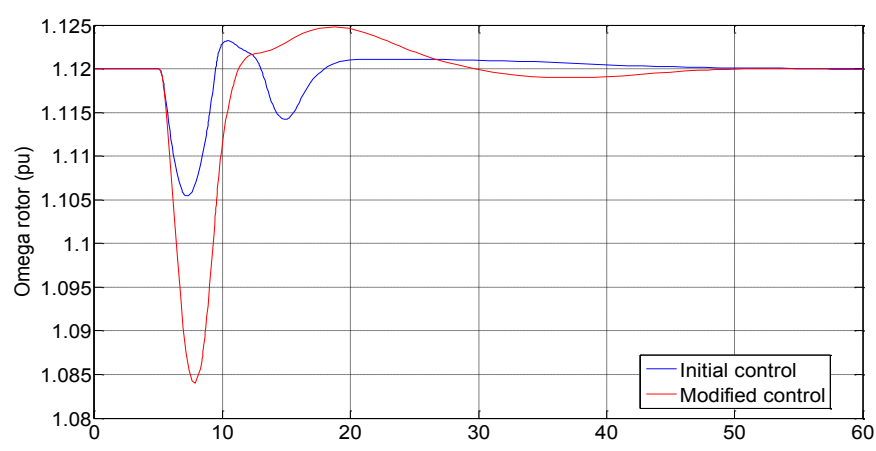

Fig. 10 DFAG rotor speed with initial and modified torque control

As can be seen in Fig. 11, the nadir of the system frequency is practically the same for the ideal inertia emulator and the modified tuning of the DFAG speed controller. It is noted that the overshoot of the frequency after $10 \mathrm{~s}$ is smaller for the case with the modified speed controller than the case without WTG dynamics. This is caused by the drop of the DFAG active power below the initial point almost simultaneously with the frequency overshoot and it cannot be drawn as a general conclusion.

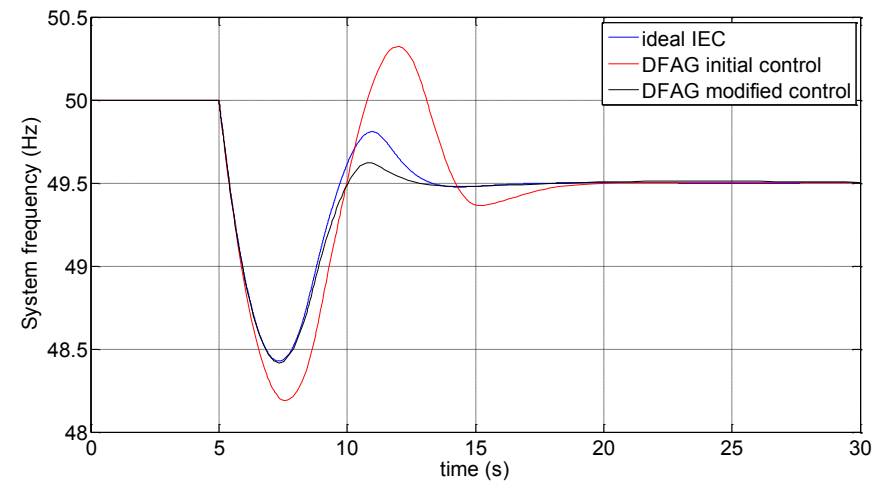

Fig. 11 Comparison of system frequency responses for initial and new speed control parameters and ideal inertia emulator

\subsection{DFAG model for initial pitch angle $\beta>0$}

In this paragraph, the wind turbine is set to operate on nominal load with pitch controller limiting the speed of the wind turbine rotor. Thus the coordination between the inertia emu- lator (with the modified tuning of the speed controller) and the pitch controller is examined.

Three operating points for different wind speeds corresponding to pitch angle $\beta=5^{\circ}, 15^{\circ}$ and $25^{\circ}$ are simulated. It was found that the effect on the frequency response was negligible. However, the effect on the wind turbine rotor speed is significant and leads to unacceptable oscillations with small damping ratio for the operating point with large pitch angle $\left(\beta=25^{\circ}\right.$ in Fig. 12).

In order to counteract this effect, the values of the gains of the pitch-control PI controller (see Fig. 8) are modified. The proportional gain is reduced to $k_{p p}=120$ from $k_{p p}=150$ and the integral gain to $k_{i p}=20$ from $k_{i p}=25$. Fig. 13 shows the response of the rotor speed for $\beta=25^{\circ}$ after this modification, where it can be seen that the damping ratio of the oscillations has significantly increased.

It is noted that the maximum power output of the DFAG model is set to $112 \%$ of the nominal power. Therefore, the inertia emulation support is not affected when the wind turbine operates on nominal load.

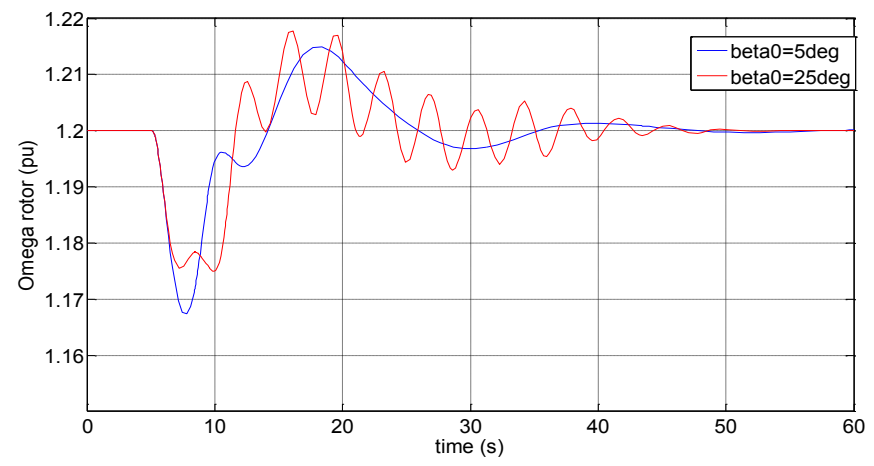

Fig. 12 DFAG rotor speed for different initial pitch angle $\beta$

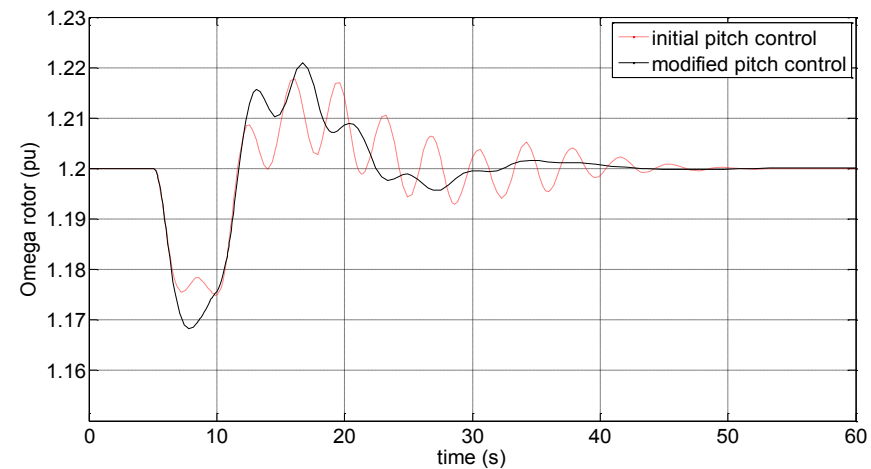

Fig. 13 DFAG rotor speed for $\beta=25^{\circ}$ after modification of the pitch controller gains

\subsection{FCWG model $(\beta=0)$}

The DFAG model of the previous paragraph is replaced here by a FCWG model. The process to determine the value of the emulator gain $k$ is the same as in the previous cases and is omitted. The proposed values of the inertia emulator parameters for the cases considered are summed up in Table 4 where it can be seen that the values are similar in all cases. 
Table 4 - Proposed inertia emulator values

\begin{tabular}{|c|c|c|c|c|}
\hline \multirow{2}{*}{ WTG System } & \multicolumn{2}{|c|}{ Fast } & \multicolumn{2}{c|}{ Slow } \\
\cline { 2 - 5 } & $\mathrm{T}_{\mathrm{w}}(\mathrm{s})$ & $\mathrm{k}(\mathrm{pu})$ & $\mathrm{T}_{\mathrm{w}}(\mathrm{s})$ & $\mathrm{k}(\mathrm{pu})$ \\
\hline no dynamics & 0.2 & 40 & 4 & 6 \\
\hline DFAG & 0.2 & 30 & 4 & 4 \\
\hline FCWG & 0.2 & 30 & 4 & 4 \\
\hline
\end{tabular}

The speed control loop of the FCWG model consists initially of only the MPPT controller (switch at position (a) in Fig.14), which based on the rotor speed $N_{r}$ introduces a power order signal $P_{\text {ord }}$ directly. In order to enhance the inertia response of the generator, the addition of a time delay before the MPPT control is examined (switch at position (b) in Fig.14). For the IEC parameter values of Table 2, it is found that a time constant of $T_{\mathrm{pc}}=1 \mathrm{~s}$ improves slightly the response of system frequency (Fig.15). The initial excursion of the frequency is drawn for comparison reasons. Larger values of the time constant do not result in any significant improvement, but cause bigger deceleration of the rotor and low-damping oscillations of the rotor speed, as shown in Fig. 16, thus they are not acceptable. Due to the large value of $T_{p c}$ in the last case (green color), the oscillation of the rotor speed is not observed in the system frequency.

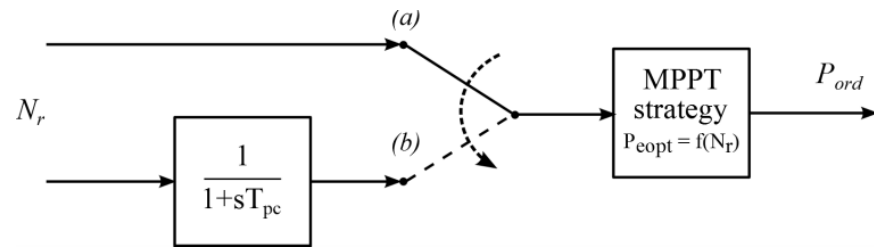

Fig. 14 FCWG speed control modification

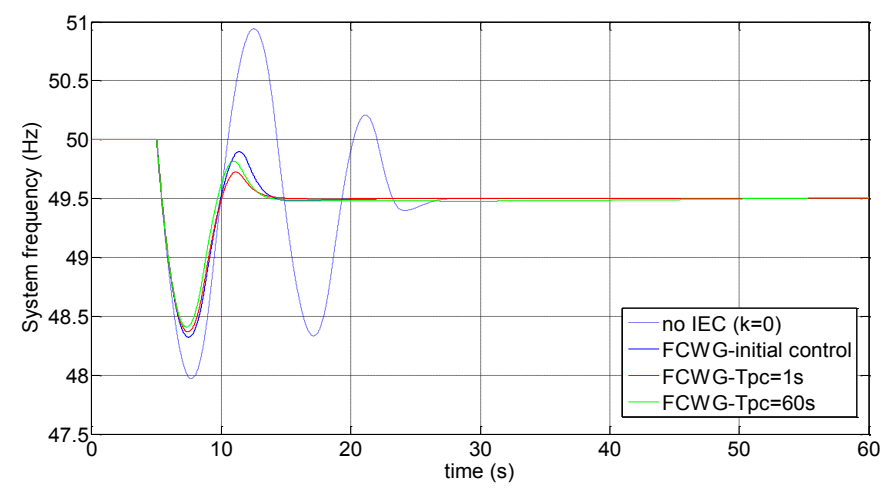

Fig. 15 Effect of time constant $T_{p c}$ to response of system frequency

\subsection{FCWG model for initial pitch angle $\beta>0$}

The same operating points as in paragraph 4.4 are simulated, i.e. $\beta=5^{\circ}, 15^{\circ}$ and $25^{\circ}$.

The effect on the response of system frequency is negligible as in the case with the DFAG model. However, there is a positive effect on the FCWG active power output, as can be seen by Fig. 17.

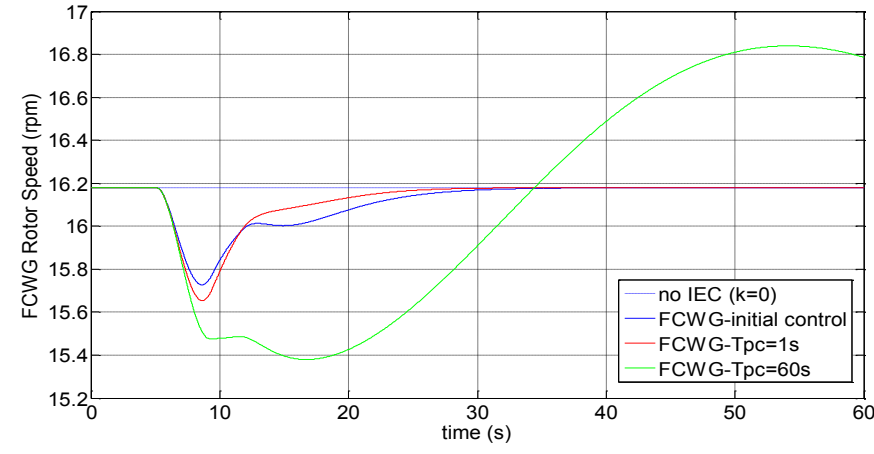

Fig. $16 \mathrm{FCWG}$ rotor speed for different values of $T_{p c}$

This happens because the deceleration of the FCWG below the nominal speed causes the pitch controller to decrease the pitch angle. This way, the aerodynamic coefficient $C_{p}$ (and thus, the aerodynamic power) increases causing less deceleration of the FCWG rotor and less drop of the active power output after the disturbance.

The active power output of Fig. 17 is limited to approximately $105 \%$ of its nominal value. This happens because of an additional limitation to the active power that the FCWG can inject to the grid and that is stricter than in the DFAG model.

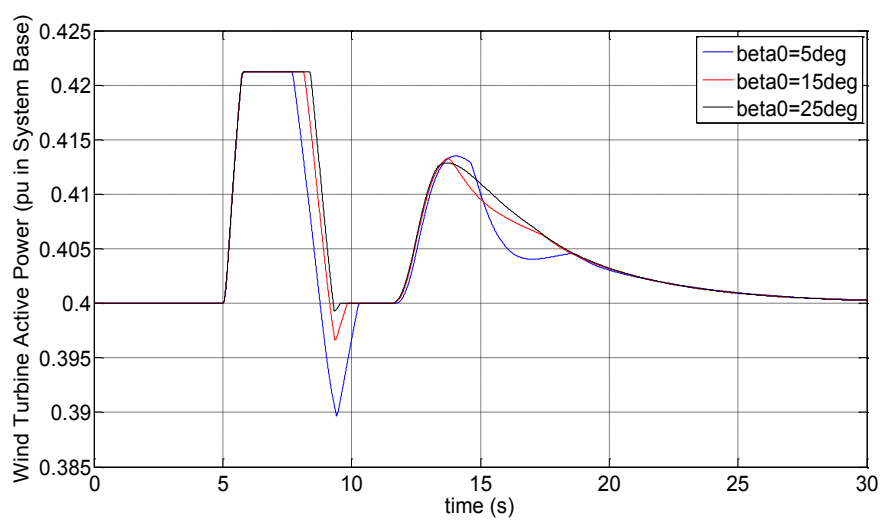

Fig.17 FCWG active power for different initial pitch angle $\beta$

\section{6: Deadband effect}

In some proposed applications of inertial emulators (for instance Ruttledge et al., 2012) a deadband is inserted before the input of the IEC, in order to avoid activation of the IEC in everyday (non-emergency) operation. For large disturbances, the effect of the deadband is expected to be small and will not significantly influence the results of the previously described procedure. This is demonstrated in Fig. 18 which shows the frequency of the slow system with the DFAG model with the modified torque control parameters of subsection 4.2. The corresponding parameters of Table 4 are selected for the IEC.

A value of $-100 \mathrm{mHz}(49.9 \mathrm{~Hz})$ is chosen for the deadband. As seen the maximum frequency drop is only slightly influenced. The dynamic response after the initial transient is also affected. Clearly the deadband (if present) could be easily incorporated in the gain selection procedure using simulation. 


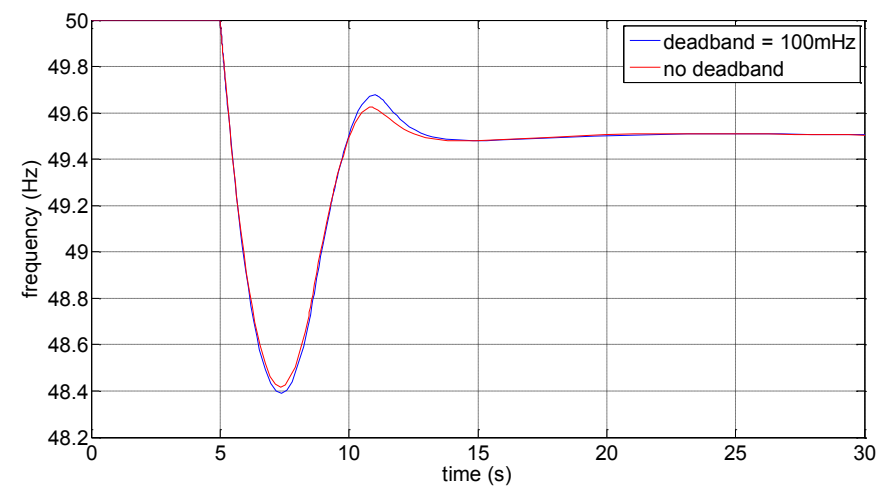

Fig. 18 Effect of deadband to system frequency (DFAG model)

\section{CONCLUSIONS}

In this paper, a design principle for wind turbine inertia emulators in an autonomous power system has been proposed. The essence of the method is to choose the time constant of the washout filter in accordance with the dominant timeconstant of the conventional units of the system. It was shown that for a system with identical units, this technique increases the inertia by a specific amount and moves the dominant open loop pole to the left of the complex plane, enhancing the stability and performance of the system.

Following the selection of the filter time constant, the gain of the inertia emulator is chosen based on simulation, so as to achieve the best possible response taking into account the nonlinearities and in particular the limiter on the inertia emulator output. The method was tested in simple systems with two types of conventional units (fast and slow) and for two different variable-speed WTG technologies. The design results were similar and frequency stability of the system was significantly enhanced in all cases. For both the DFAG and FCWG models some adjustments of the active power control loops were necessary to improve system performance with the IEC.
Clearly, this method has to be fine tuned for specific system applications. However, the design principles proposed give promising results and the key problems associated with possible interactions with other WTG controls have been highlighted. Future research will focus in coordinating the response of WTG inertia emulator with underfrequency protection of an autonomous system.

\section{REFERENCES}

Gautam D., Goel L., Ayyanar R., Vittal V., Harbour T. (2011) "Control Strategy to Mitigate the Impact of Reduced Inertia Due to Doubly Fed Induction Generators on Large Power Systems", IEEE Transactions on Power Systems, Feb. 2011, 26(1) 214224.

Lalor G., Ritchie J., Rourke S., Flynn D., O’ Malley M.J., "Dynamic Frequency Control with Increasing Wind Generation", Power Engineering Society General Meeting, June 2004, Vol. 2, 17151720.

Lalor G., Mullane A., O' Malley M.J., "Frequency Control and wind turbine technologies", IEEE Transactions on Power Systems, Nov. 2005, 20(4), 1905-1913.

Marinelli M., Massucco S., Mansoldo A., Norton M., "Analysis of Inertial Response and Primary Power-Frequency Control Provision by Doubly Fed Induction Generator Wind Turbines in a small Power System", Proceedings of the 17th Power Systems Computation Conference, 2011, Stockholm, Sweden.

Morren J., de Haan Sjoerd W.H., Kling W.L., Ferreira J.A., "Wind Turbines Emulating Inertia and Supporting Primary Frequency Control", IEEE Transactions on Power Systems, Feb. 2006, 21(1), 433-434.

Nanou S.I., Tsourakis G., Vournas C.D., "Full Converter wind generator modelling for transient stability studies", PowerTech, 2011 IEEE Trondheim, 19-23 June 2011, 1-7.

Ruttledge L., Miller, N. W., O'Sullivan, J., Flynn, D., "Frequency Response of Power Systems with Variable Speed Wind Turbines", IEEE Trans. on Sustainable Energy, Oct. 2012, 3(4), 683-691.

Tsourakis G., Nomikos B.M., Vournas C.D., "Effect of wind parks with doubly fed asynchronous generators on small-signal stability”, Electric Power Systems Research, 2009, 79(1), 190-200.

Vournas C.D., "Unstable Frequency Oscillations in a SlowResponse Reheat-Turbine Generator", IEEE Power Engineering Society Winter Meeting, Jan 1999, 140-144. 